\title{
Teaching Design in Computer Engineering
}

\author{
Ken Ferens \\ Dept. of Electrical and Computer Engineering \\ University of Manitoba, Winnipeg, MB, Canada \\ Ken.Ferens@ad.umanitoba.ca
}

Abstract-This paper reports on a project based learning approach taken to teach the ECE 3740 Systems Engineering Principles and ECE 3730 Principles of Embedded Systems Design courses at the University of Manitoba. These courses were 100\% hands-on, and each student was given development hardware and software in a lunch box to take home and work on projects throughout the course. Industry representative projects were chosen based on the author's 5 years of experience working in the embedded systems industry. The courses were given in a company-like setting, where the lectures and laboratories were organized as product requirements gathering and analysis, design modeling and review, test plan and procedures, engineering change request and management, documentation, and product deployment meetings and events. The test and final exam were performed by students in the laboratory; they brought their embedded systems hardware in the lunch box, solved the given hands-on problems of the test/exam, and demonstrated their solutions, in real-time. This novel methodology allowed the examiner to directly assess student performance in the CEAB attributes of Design, Analysis, Investigation, and Tools, because their designs and solutions were actually demonstrated in actual hardware and software, not just on paper, like the conventional assessment approach for tests and exams.

Keywords - design, creativity, design thinking, project based learning, CEAB attributes.

\section{INTRODUCTION}

Teaching design to computer engineering students is difficult because of a lack of a unified understanding and representation. There are many different terms that are used interchangeably to mean the same thing in regards to design. For instance, consider a target project (aka abstract idea, problem, or product). Many representatives from industry and academia refer to the intended (desired) functions (behavior) of the target as requirements, specifications, or objectives. Moreover, when the physical realization must be implemented in a certain way, this is sometimes referred to as "constraints on the design," while others refer to this as "design requirements." Some people do not regard a difference between the meaning of the terms requirements and design. Furthermore, the process of design varies greatly as found by [1]. There is no single universally agreed on standard process, even within a single discipline, like computer engineering. Sure, there are many different kinds of standard activities, development processes, and methodologies, such as for software engineering [2], but the lack of a single standard makes design difficult to teach and learn.

There are no standards for guiding designers when making decisions. The research performed by [3] and others have attempted to qualify and quantify design and its processes through mathematical formulation and modeling, such as through decision and economic theories. When a designer is faced with making a design choice, a specific alternative is typically chosen based mostly on a subjective evaluation of how well the alternative meets the requirements. This decision could be modeled using fuzzy set theory, artificial neural networks, swarm optimization techniques, or other heuristic methods, but, there is no universal agreement. Furthermore, given the differences in details between different disciplines within Engineering, a mathematical model of design would be limited to vague and high level guidelines.

How to teaching students to be creative and how to think about design are not well understood, either. The creativity aspect is perhaps the most elusive component in design. Design is the physical realization of an abstract idea, and creativity can be measured as the degree of novelty of the realization. A design is said to be "good" if it satisfies the desired requirements of the idea, independent of whether the design is creative or not. Can people learn to be creative, or is the degree of creativity burnt into a person's genes, something which cannot be 
altered in the present body? If creativity can be learned, then what is the process which goes on in the mind when a person creates a solution to a problem? Can we teach that? Can we strengthen, enhance, and build upon that process? Teaching design is difficult.

Many engineering education researchers [1,4] report that teaching design is difficult, due to: inadequate understanding of design thinking and what goes on in a design expert's mind during the design process; complexity of the target system; the processes, protocols, and communication involved in designing in teams; inadequate assessment procedures; observing and minimizing environmental and social impacts, and due to the plethora of design languages. For instance, typical engineering curricula assessment methodologies limit the extent to which they can measure design ability. According to [4], many engineering examination questions require that students provide a unique and correct answer to a given problem (convergent questioning), and that the examination questions do not assess a student's ability to generate new questions given a seed question (divergent questioning). A concrete example of this might be that in the examination world, students are penalized if they change the question to suit their solution. But, different design alternatives might not be reachable if the original question is made fixed and immutable. Therefore, in the real world, some design alternatives can only be made possible by changing the requirements (i.e., seed question), and these alternatives may turn out to be better designs.

Many researchers [1], greedy algorithms, and common sense suggest that people with design experience should teach design to students. Experienced designers in industry have a proven record, and are generally regarded as experts in the field. Is there a global better knowledge base about design than that known locally? Probably, yes. However, if academics have not found the global optimal solution to teaching design at this point in time, then the practitioners in industry, who have learned how to do design well by doing and practising within their local world, would represent the best choice.

This paper takes a hybrid approach to proposing methodologies for teaching design. An academic instructor with industry design experience is arguably in the best position to teach design to students. As an industry experienced designer and leader, the instructor can instill best industry practises to students, and thus better prepare them for work in industry. As an academic, particularly involved with the betterment of engineering education, the instructor can in addition provide students with background academic knowledge required for design.
Furthermore, this paper employs a project-basedlearning and hands-on approach to teaching design. As an experienced designer from the industry, the instructor would know which projects to select for teaching: proposing industry representative projects that would provide students with exposure to real world problems and their solutions. Currently, research data on the effectiveness of an industry representative with academic background is not readily available.

This leads to the next topic, which is: how shall the effectiveness be measured? Many open loop systems exist, and they operate fairly well, but open loop assessment method may not be accepted by peer reviewers and the System. Alternatively, assessment methodologies which attempt to measure student performance before and after application of the "medicine" are fraught with errors, due to the many uncontrolled variables. Instead, this paper takes the approach of consulting with industry. Students are like an output of a system, which is fed into industry. Over $90 \%$ of students proceed to work in industry. Therefore, industry is the direct sink of an engineering program's output, i.e., their graduates. This paper proposes to use industry forums [5] to both inform industry members of the new approaches to teaching design, and also to obtain their opinions to any perceived differences between new graduates continually and over the span of many years.

\section{Project BASED LEARNING}

In the fall semester of 2012, a project-based learning (PBL) and 100\% hands-on learning approach was implemented in the course "ECE 3740 Systems Engineering Principles" in the Department of Electrical and Computer Engineering, University of Manitoba. The overarching goal of the course project was to implement a wireless sensor network for gathering sensor readings and reporting the data to a central base station on the Internet. This project was intended as a template of many possible applications, such as a real-time vending machine monitoring system (people can monitor the inventory of a vending machine using a web browser anywhere on the Internet); real-time smart building monitoring and control system (people can monitor and control physical parameters (such as temperature, lights, and door locks) of the environment within a building using a web browser anywhere on the Internet); and shopping for dummies (wireless nodes communicate prices and information about products in department stores to shoppers' smart phones, which, in turn, communicate with a service oriented architecture based server to obtain and store location with the best prices). 
The goals of the PBL course were to provide students with an opportunity to gain experience with the design principles of divide and conquer; incremental design; minimizing complexity; maximizing cohesion; unit and system test plans and procedures; UML modeling; multitasking; and C, HTML, and AJAX code development. These concepts were not taught to students through the lecture style of teaching; rather, students learned these concepts on their own by experiencing them when and if needed throughout the design and development process. While students were experiencing these issues, lectures were given to guide, strengthen, and reinforce the concepts. Anecdotal observations showed a higher level of learning, participation, and retention in students, when they received a lecture on a specific concept after having experiencing that concept. During those lectures, students asked more detailed and relevant questions. The feedback was wonderful and invigorating.

The PBL and hands-on approach to teaching design demands a huge cost in terms of time, resources and funding. The instructor must go through all of the development steps with the students, like a student. A benefit of this process is that the instructor becom3es very familiar with the abilities of each student, and can witness their academic development throughout the course. Grading the projects, tests, and final exam is extremely difficult due to the design nature and the many possible different variations of the solutions. The course instructor spent two months during the summer of 2012 preparing for the fall course ECE 3740 . The MX7cK board from Digilent [6] was obtained. This board has several nice features, such as $80 \mathrm{MHz}$ 32-bit microcontroller, 10/100 Ethernet controller, USB controller, CAN controllers, SPI interface, $\mathrm{I}^{2} \mathrm{C}$ interface, and many others. Expansion connectors exist on the board to add peripherals, Pmods, such as WiFi Interface, temperature sensor, gyroscope, and accelerometer. Funding for the hands-on equipment for the ECE 3730 and ECE 3740 courses was obtained from the Design Engineering Department [7], and the Department of Electrical and Computer Engineering, the Faculty of Engineering, University of Manitoba.

The MX7cK reference manual states that "The Microchip Applications Library, available for download from the Microchip web site provides full protocol stack support compatible with the PIC32MX795 MAC and the LAN8720 PHY." The author learned there were several modifications required. Specific details and a list of instructions to implement the necessary changes may be viewed from [8]. Furthermore, the MX7cK board uses an $I^{2} \mathrm{C}$ EEPROM memory, while the Microchip stack software assumes an SPI EEPROM. The EEPROM is needed to store web pages on the server. The author rewrote the SPI EEPROM code so that it would work with the $\mathrm{I}^{2} \mathrm{C}$ EEPROM on the MX7cK board. Specific details of the conversion and $\mathrm{C}$-code can be obtained from [8].

The overall ECE 3740 course project was split into five smaller projects, and each project was further split into three parts (Pre-assignment, lab, and postassignment). Splitting the course project into smaller parts demonstrated to students how to practise divide and conquer, incremental development, unit testing, minimization of complexity, and maximization of cohesion. Since students implemented the sub-projects, they became involved in the procedure, and were able to more deeply understand how and why the project was broken down into smaller pieces. This was an example of how an industry leader can guide students in how to think about design and how to implement a design process.

The Systems Engineering Principles Project 1 (SEP Project 1) was implemented by the series Assignment 1, Laboratory 1, and Assignment 2. The SEP Project 1 had two main objectives: the first objective was to gain experience in using the Microchip Integrated Development Environment (IDE) and the MX7cK board. Students also became familiar with the procedures involved in developing TCP/IP Stack applications; the second objective was to design $\mathrm{C}$ programs to control basic peripherals on the MX7cK board. Specifically, Lab 1 and Assignment 2 required that students design a console control of the LEDs and push buttons, using a telnet client on the host to connect to their TCP code on the MX7cK board.

The Systems Engineering Principles Project 2 (SEP Project 2) was implemented by the series Assignment 3, Laboratory 2, and Assignment 4. Assignment 3 required that students design an LED Rotate Server, and test this with a remote telnet client. Laboratory 2 expanded on this by requiring another $\mathrm{MX} 7 \mathrm{cK}$ board act as a TCP client and connecting to the TCP TED Rotate Server. During the development students were confronted with a problem regarding how an autonomous client (i.e., MX7cK board) can know if a Rotate Server is available on the network. Laboratory 2 used a fixed IP address server, while Assignment 4 required the autonomous client to learn of the presence of a Rotate Server through a dynamic IP address protocol, which students designed. Being confronted with this issue during development, students were intimately involved with the issues.

The Systems Engineering Principles Project 3 (SEP Project 3) was implemented by the series Assignment 5, Laboratory 3, and Assignment 6. The objective of SEP Project 3 was to allow a user operating a web browser to view the status of the push button switches and the status 
of the LEDs on any MX7cK board on a network. Also, the system should allow a user operating a web browser to set the LEDs on/off on any MX7cK board. This project constrains the design as shown in Fig. 1, i.e., due to network security issues, the syste $4 \mathrm{~m}$ was to be implemented on local area network (LAN). Assignment 5 was to update the TCP/IP Stack software to use the $I^{2} \mathrm{C}$ EEPROM on the MX7cK board, and to unit-test several basic http server functions.

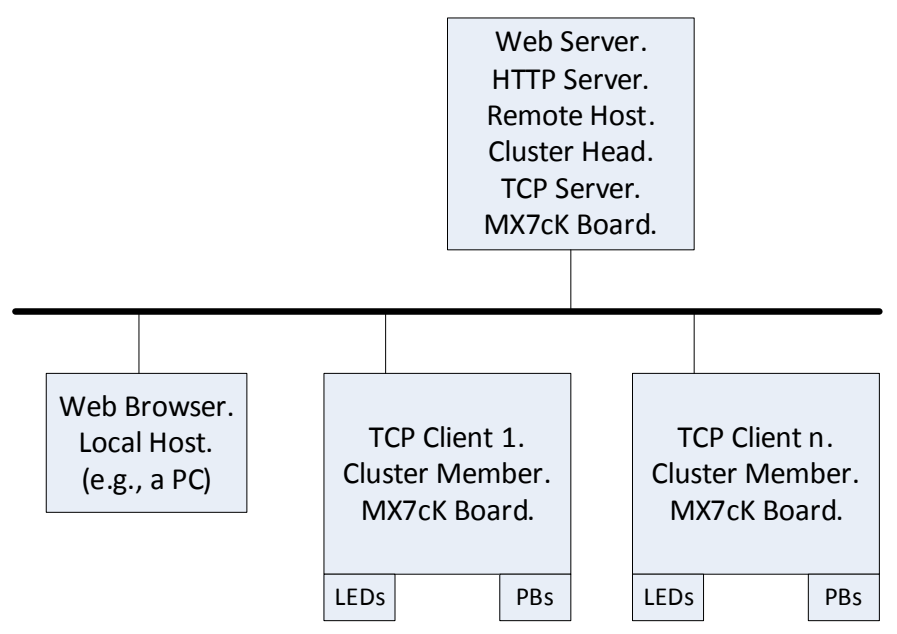

Fig. 1 Network architecture for Project 3.

Laboratory 3 required that students design a system that allows multiple client $\mathrm{MX} 7 \mathrm{cK}$ boards (cluster members) to make TCP connections to another MX7cK board acting as the server (cluster head). A client board sent its push button and LED status to the server periodically. Also, the server sent commands to set the LEDs on/off, periodically. Students learned they needed to develop a communications protocol between the server and clients, such as for clients sending update messages to the server, and the server sending commands to the clients. During this development, students experienced the concept of client-server architecture, and lectures on that topic were given simultaneously to reinforce the concept.

Assignment 6 required students to design a system to allow a user operating a web browser on a host computer to view the status of the Push Button switches and the status of the LEDs on any MX7cK board. Also, the system allowed a user operating a web browser to set the LEDs on/off on any MX7cK board. Students developed a web site and uploaded the code onto the MX7cK board. Students wrote C-code on the http server to implement functions such as gathering LED and push button status on the server board (cluster head) and remotely connected MX7cK nodes (cluster members), and reporting this information to the web page on the users web browser.
Students wrote C-code, HTML, and AJAX (Asynchronous Java and XML) to implement these functions. Students also developed communications protocols between the user's web page, the http server, and nodes (cluster members). By developing web pages and uploading them to their http server on the MX7cK board, students experienced the HTTP Client-Server paradigm. Lectures on these topics were given contemporaneously to secure the concepts.

The Systems Engineering Principles Project 4 (SEP Project 4) was implemented by the series Assignment 7, Laboratory 4, and Assignment 8. Each part required students to develop simple applications for implementing several different types Pmod sensors, including microphone, temperature, gyroscope, and accelerometer. With these sensors, each MX7cK board was now equipped with LEDs, push button switches, and microphone, temperature, gyroscope, and accelerometer sensors. During this development process students experienced the concept of changing requirements. In particular, the communications protocol and code previously developed for communicating LED and Push Button status and commands needed to be changed to include these additional sensors. Due to this particular experience, students learned that their developments should include hooks and room to allow future extensions and features. For example, the number of bytes used to encode a command should be sufficient to allow for future expansion.

The Systems Engineering Principles Project 5 (SEP Project 5) was implemented by the series Assignment 9, Laboratory 4, and Assignment 10.

Assignment 9 developed a clustering process for the MX7cK boards. As students learned, when WSN nodes are deployed in inaccessible areas where there is no grid power, each board may be powered by batteries. Consequently, all radio communications and processing tasks must be energy aware in order to preserve power and maintain the life of a node and the network application. The communications protocol previously developed was revisited and redesigned to minimize the amount of messages sent, since radio transmission expend the most amount of energy. The students ran a brainstorming session during class time to produce design alternatives. They found the best alternative was to organize the MX7cK boards into clusters of nodes, where each node was a cluster member, and one node was elected cluster member (Fig. 2). Students designed and wrote $\mathrm{C}$-code to implement the cluster formation process. To practise their modeling, communication, and visualization skills, students drew UML state diagrams to explain the clustering process (Fig. 3). 
Cluster head elections were performed initially, and whenever a current cluster head's residual energy dropped below a certain threshold. Nodes participating in the cluster head election process start a timer, which is inversely proportional to the residual energy of the node. If a node's timer expires and the node has not received a cluster head declaration message, then that node declares itself cluster head. Each node receiving a cluster head declaration message becomes a cluster member of that cluster head.

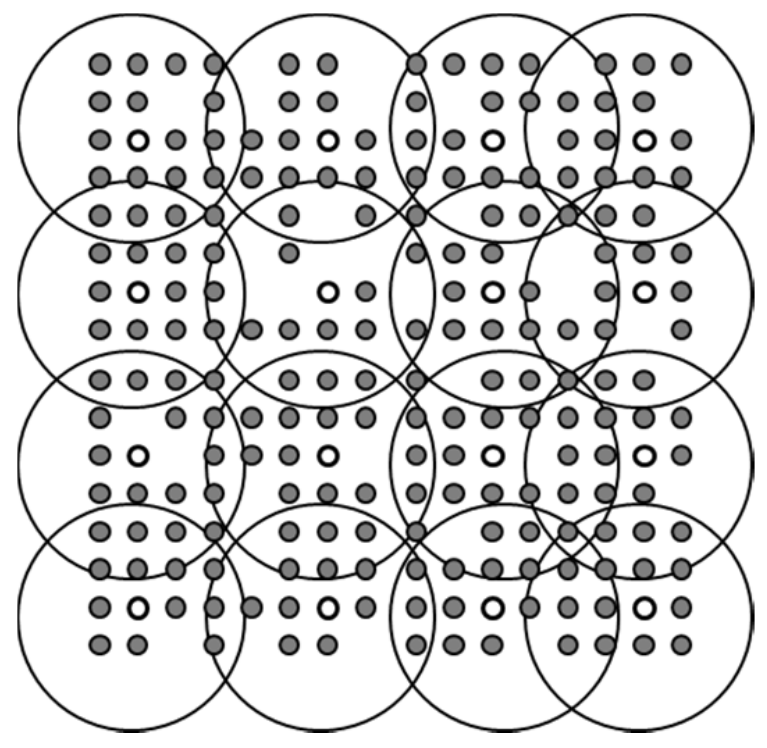

Fig. 2 Clusters of MX7cK boards.

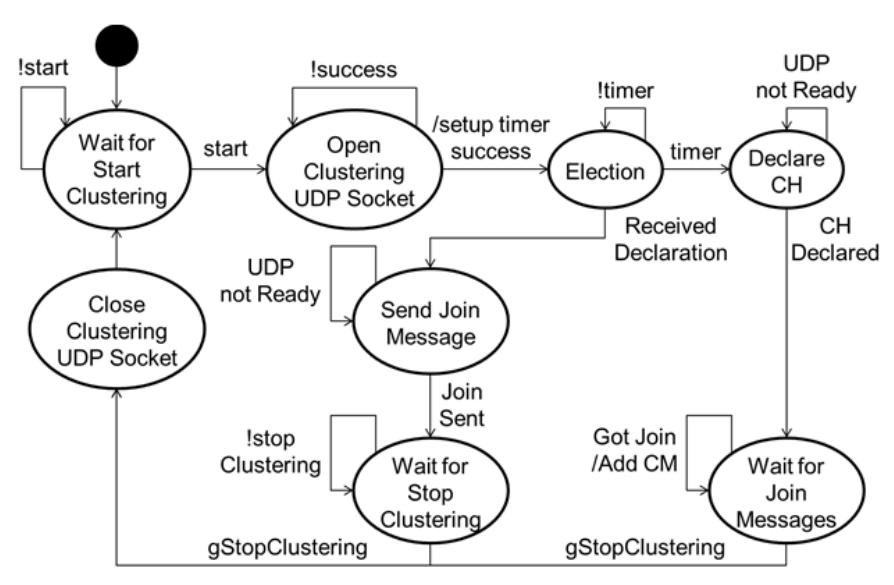

Fig. 3 State diagram of the clustering process.

Cluster members would send their sensor readings to the cluster head according to a Time Division Multiple Access TDMA schedule, which was created by the cluster head (see Fig. 4 for an example).
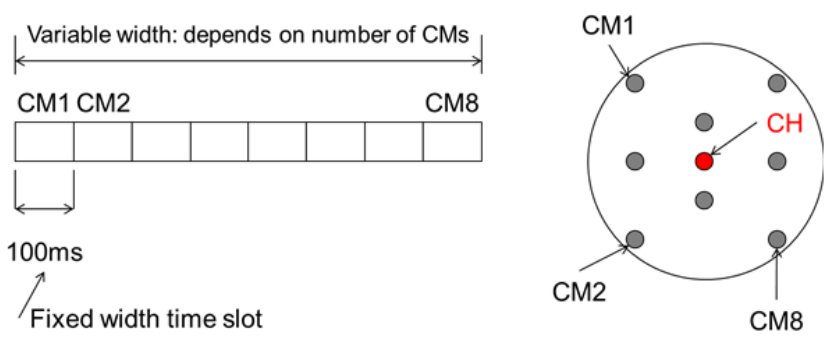

Fig. 4 TDMA schedule format.

Laboratory 5 required students develop code for each cluster member to send its readings to the cluster head. Finally, assignment 10 required students to develop code to integrate the system components, which were previously incrementally developed. The integrated system became a self-configuring, self-healing, loadbalanced wireless sensor network, with http server. Once a MX7cK node is deployed in the field (e.g., placed at some remote location), it automatically interacts with any other node in radio range to engage in the clustering process, sensing, and monitoring and reporting of sensor readings. The cluster head automatically aggregates sensor readings, creates and disseminates TDMA schedules for its cluster members, and interacts with other cluster members for transmitting data to the master cluster head (i.e., the http server). Actors (i.e., users) interact with the system through a custom made web site. The web site offers the ability to select a cluster of nodes or a cluster member of a particular cluster. A user can select nodes for configuration, e.g., calibrating the sensors, or for status and monitoring purposes. Thus, a scientist (or anyone) anywhere on the Internet may open the ECE 3740 web site and obtain real-time monitoring and control capability of any number of $\mathrm{MX} 7 \mathrm{cK}$ boards deployed in the field.

\section{SUMMARY}

This paper reports on the experiences gained in applying a project-based-learning and 100\% hands-on approach to teaching computer engineering courses at the University of Manitoba. For the ECE 3740 course, the main goal of the course project was to implement a wireless sensor network for gathering sensor readings at a remote location, and reporting the data to a central base station on the Internet. This project was intended as a template of many possible applications, such as a realtime vending machine monitoring system; real-time smart building monitoring and control system; and shopping for dummies. An industry experienced academic instructor guided students through the design and development 
process. Anecdotal observations suggested that students enjoyed the course much more than in previous years. Students also appreciated the PBL approach, as it gave them hands-on experience and opportunity to design and develop an embedded system that was industrially relevant. A student who took the course in a past year sat in the new course just because he heard about the handson approach and wanted to experience it firsthand. The instructor noted that due to the course-long one-on-one interaction with students, in the 19 years teaching embedded systems related courses, he has never been surer of the grades awarded to students for their performance in the course. This novel methodology allowed the examiner to directly assess student performance in the CEAB attributes of Design, Analysis, Investigation, and Tools, because their designs and solutions were actually demonstrated in actual hardware and software, not just on paper, like the conventional approach to student tests/exams. An incredible amount of time, resources and funding was required to successfully implement this PBL and hands-on course. But, it was worthwhile.

\section{ACKNOWLEDGEMENTS}

The author acknowledges Dr. Douglas W. Ruth and the Department of Design Engineering for funding the hardware for ECE 3730, as well as supporting the projectbased-learning and hands-on approach to teaching computer engineering courses in the Department of Electrical and Computer Engineering, University of Manitoba.

\section{REFERENCES}

[1] Rim Razzouk and Valerie Shute, "What Is Design Thinking and Why Is It Important?," Review of Educational Research, vol. 82, no. 3, pp. 330-348, September 2012.
[2] Wikipedia. (2013, April) Software development process - Wikipedia, the free encyclopedia. [Online]. http://en.wikipedia.org/wiki/Software development process

[3] G. A. Hazelrigg, "An Axiomatic Framework for Engineering Design," Journal of Mechanical Design, vol. 121, p. 3420347, 1999.

[4] Clive L. Dym, Alice M. Agogino, Azgur Eris, Daniel D. Frey, and Lary J. Leifer, "Engineering Design Thinking, Teaching, and Learning," Journal of Engineering Education, vol. 94, pp. 103-120, January 2005.

[5] Ken Ferens, "Industry Focus Group Forum Approach for Assessing Undergraduate Engineering Program Outcomes," in Proc. of the Canadian Engineering Education Association (CEEA/ACEG) Conference, St. John's, Newfoundland, 2011.

[6] Digilent Inc. (2013, January) Digilent Inc. [Online]. http://www.digilentinc.com

[7] Design Engineering, Faculty of Engineering. (2013, April) University of Manitoba - Faculty of Engineering - Desing Engineering. [Online]. http://umanitoba.ca/engineering/departments/design/

[8] Ken Ferens. (2012, September) ECE 3740 Systems Engineering Principles 1. [Online]. http://ece.eng.umanitoba.ca/undergraduate/ECE3740 I

[9] Digilent Inc. (2012, January) Cerebott MX7cK ${ }^{\mathrm{TM}}$ Board Reference Manual. [Online]. http://www.digilentinc.com/Products/Detail.cfm?Na vPath $=2,396,986 \&$ Prod $=$ CEREBOT-MX7CK

[10] SMSC. (2012, August) SMSC - Product Information. [Online]. http://www.smsc.com/media/Downloads Public/Dat a Sheets/8720a.pdf

[11] Digilent Inc. (2011, August) Cerebott MX7cK $\mathrm{cK}^{\mathrm{TM}}$ Board Schematics. [Online]. http://www.digilentinc.com/Products/Detail.cfm?Na vPath $=2,396,986 \&$ Prod=CEREBOT-MX7CK 